\title{
Enhancing cultural competence: Trans-Atlantic experiences of European and Canadian nursing students
}

\author{
Liisa Koskinen RN PhD \\ •., Savonia University of Applied Sciences, Unit of Health, Kuopio, Finland \\ Barbara Campbell RN PhD \\ $\bullet$, University of Prince Edward Island, Charlottetown, Prince Edward Island, Canada \\ Clara Aarts RN RCN PhD \\ •, Uppsala University, Department of Public Health and Caring Sciences, Uppsala, Sweden \\ France Chassé MScN PhD \\ •, University of Moncton, School of Nursing, Edmundston Campus, Edmundston, New Brunswick, Canada \\ Ann Hemingway RN PhD \\ ••, Bournemouth University, School of Health and Social Care, Bournemouth, Dorset, UK \\ Tiina Juhansoo MD PhD \\ $\bullet$, Tallinn Health College, Tallinn, Estonia \\ Maureen P Mitchell RN MN EdD \\ •, Mount Royal College, School of Nursing, Calgary, Alberta, Canada \\ France $\mathrm{L}$ Marquis MScInf \\ ••, University of Moncton, School of Nursing, Edmundston Campus, Edmundston, New Brunswick, Canada \\ Kim A Critcley RN PhD \\ $\bullet$, University of Prince Edward Island, Charlottetown, Prince Edward Island, Canada

\section{Pamela M Nordstrom RN PhD} \\ •-, Mount Royal College, School of Nursing, Calgary, Alberta, Canada \\ Accepted for publication June 2009
}

Koskinen L, Campbell B, Aarts C, Chassé F, Hemingway A, Juhansoo T, Mitchell MP, Marquis FL, Critcley KA, Nordstrom PM. International Journal of Nursing Practice 2009; ••: ••_••

Correspondence: Liisa Koskinen, Savonia University of Applied Sciences, Unit of Health Care, PL 1028, 70111 Kuopio, Finland. Email: liisa.koskinen@savonia.fi 
Enhancing cultural competence: Trans-Atlantic experiences of European and Canadian nursing students

This paper describes the enhancement of cultural competence through trans-Atlantic rural community experiences of European and Canadian nursing students using critical incident technique (CIT) as the students' reflective writing method. The data generated from 48 students' recordings about 134 critical incidents over a 2-year project were analysed by qualitative content analysis. Five main learning categories were identified as: cross-cultural ethical issues; cultural and social differences; health-care inequalities; population health concerns; and personal and professional awareness. Four emergent cultural perspectives for the health sector that became apparent from the reflections were: health promotion realm; sensitivity to social and cultural aspects of people's lives; channels between the health sector and society; cultural language and stories of local people. CIT was successfully used to foster European and Canadian undergraduate students' cultural reflections resulting in considerations and suggestions for future endeavours to enhance cultural competence in nursing education.

Key words: community nursing, critical incident, cultural competence, trans-Atlantic exchange.

Nursing education worldwide needs to respond to the challenges of an increasingly more diverse society by developing study programmes that educate culturally competent practitioners. Trans-Atlantic student exchange projects between universities from two continents, as described in this article, have become important methods for advancing cultural competence inherent in nursing education. During the cross-cultural exchange, students are immersed into the foreign culture and language over an extended period of time, offering them a potential opportunity for further development of cultural competence. ${ }^{1}$

Cultural competence is regarded as an ongoing personal maturation process that includes increasing self-awareness, ability to see through others' eyes when conflicting values and expectations occur in interaction, willingness to negotiate mutually acceptable solutions and capacity to act in culturally diverse contexts. ${ }^{2}$ Culturally competent nursing care can be defined as the sensitivity towards diversity in other people including professional advocacy for human rights and advancement of equal rights for all needing help. ${ }^{3,4}$ This definition, which considers cultural competence as the personal and collective responsibility towards other people, was adapted as the framework for our project.

Cultural experiences can be provided to learners through diverse experiential methods in real or simulated cultural contexts, ${ }^{5}$ but learning cultural competence requires an extended cultural immersion including encounters with culturally different people. ${ }^{6,7}$ In this research project, senior nursing students from universities in four European countries (England, Estonia, Finland,
Sweden) and three Canadian universities (Alberta, New Brunswick, Prince Edward Island) participated in a transAtlantic exchange during 8-16 weeks. The project was carried out in 2004-2007 and aimed to foster a transAtlantic student exchanges in the spirit of the Ottawa Charter declaration. ${ }^{8}$ The goal of the project was to strengthen participants' awareness of community cultural and social inequalities in health and well-being.

Participating students practised in various rural community placements as cross-cultural student nurses, mentored by the local nurses and supervised by the teaching staff of the host university. The students were asked to reflect their cultural experiences from their foreign stay by writing reflective journals using critical incident technique (CIT). During the past 10 years, the CIT, developed by Dr John C. Flanagan in 1950s, ${ }^{9}$ has been used increasingly in nursing education as a method to advance critical thinking and reflection skills ${ }^{10}$ and to teach cultural awareness. ${ }^{11}$

A critical incident can be described as an occurrence that makes a significant contribution, either positively or negatively, to an activity or phenomenon. According to Brookfield, critical incidents are brief descriptions written by learners about meaningful events in their lives. ${ }^{12}$ By reflectively writing the chosen critical experience, students might become more aware of their own values, emotions, reactions and the personal impact of the event at hand. In health care, the particular strengths of this approach is its potential for encouraging reflection on the emotions arising from complicated care situations and thus supporting the identification and subsequent handling of any emotional burden experienced. ${ }^{13,14}$ 
It is essential that nursing students' critical thinking and reflection skills are challenged during their education enabling them to make an informed decision in rapidly changing health-care situations with clients from various cultural backgrounds. It is also essential that students learn to recognize and articulate the feelings and emotions connected with diverse cultural encounters. Therefore, it seems that developing a greater understanding of the use of the CIT method in a cross-cultural learning environment would be valuable.

\section{THE STUDY} Aim

The aim of this study was to explore the enhancement of cultural competence of European and Canadian undergraduate nursing students during their trans-Atlantic rural community placement by recognizing the cultural and social inequalities in the host country.

\section{Participants}

Altogether, 48 European $(n=21)$ and Canadian $(n=27)$ students were involved in the project. They were introduced to the overseas host partners, the general content of the project, and engaged in the concrete exchange orientation including the use of CIT as a writing method. The students were acquainted with the web-based learning 7 management system (Blackboard(C) as the method to communicate with their peers in the host country. Finally the students, who travelled in a group of two or three, were immersed into the overseas exchange.

The language of teaching was English for both European and Canadian students although students were exposed to the host language in their clinical situations. The European students could speak English and were encouraged to practise conversing in English before travelling to Canada. The Canadian students could not speak Finnish, Estonian or Swedish but did receive basic language preparation in the host countries before their practicum experience.

\section{Data collection}

The data consisted of 134 critical incidents (one to two pages in length) written by 21 European and 27 Canadian undergraduate nursing students during their overseas exchange. The critical incident record sheet, designed for the purpose of this project, included a short introduction section followed by six key steps for organizing students' reflection and writing.

\section{Data analysis}

The students were asked to write and reflect in their home language resulting in original data being presented in English, Estonian, Finnish, French and Swedish. The data were analysed in two stages. In the first stage, the students' written critical incidents were analysed and translated into English in the seven home universities by two researchers from each university according to the agreed reduction guideline (Fig. 1).

In the second stage, Miles and Huberman's ${ }^{15}$ method was used to analyse the summary data received from each university partner. This method was chosen because it allows categorizing of learning experiences and identification of feelings and emotions as dimensions of learning. The focus of the analysis was in the reflections connected with the objectives of the project and with student learning in a foreign cultural context. In this stage, data analysis was carried out as a collaborative endeavour between a European and a Canadian researcher.

The analysis consisted of three simultaneously occurring steps: data reduction, data display, conclusion and verification. At the reduction step, key words and phrases related to the focus of the research question were highlighted and coded inductively. Data display included comparison of the codes with one another, clustering of similar codes into subcategories and labelling of the subcategories according to their content and meaning. The reflections of the European and Canadian students were conducted separately on the above two steps. Finally, at the conclusion and verification step, five main categories were created to describe the cross-cultural overseas learning experiences of the two student groups.

\section{FINDINGS}

\section{Cross-cultural ethical issues}

Students' reflections from the trans-Atlantic experiences included various cross-cultural ethical issues. The reflections highlighted students' learning by witnessing care situations where they had to grapple with personal ethical principles that interfered with their moral values, beliefs, communication abilities and interpretation of professionalism when delivering nursing care. The cross-cultural ethical issues related to inappropriateness in nursing care, stressful nurse-patient interaction, inequality in service and concerns arising from sexual health practice.

The cross-cultural ethical issues were reflected by both student groups but there were slight cultural differences in what the students regarded as an ethically critical 
Your role is to submit a 2-3 page summary of yourreview of students' critical incidents. The focus of this research is about how the students reflected their critical incidents (3/student) and what they learned from them. Please collate your findings from all the papers into one response to each question. You should also have a colleague verify your analysis, to strengthen reliability.

- Number of critical incidents - How many incidents (from how many students) did you analyse?

- Type of critical incidents - What the critical incidents were?

- Reason why the incidents were critical-Why the students viewed the incidents as critical?

- Focus of the project-How the students reflected the critical incidents in relation to inequalities question/Ottawa Charter/cultural aspects of the experience/own global awareness?

- Students' learning-What did the students say they learned from their incidents?

- Implications for Nursing-How did the students reflect their own role as student nurses in particular in relation to their role in health promotion?

- Implications for Nursing Education-What learning is there for you as an instructor?

- Quality of the incidents?-Did the incidents demonstrate reflection? Did the students use references? What did they write about their emotions and feelings?

Figure 1. The data reduction guideline for the first stage of data analysis. experience. The Canadian students reflected more concerns while in Europe than their European colleagues did while in Canada. Issues included a perceived ignorance of nursing principles such as protection of patient privacy, concerns about patient rights for informed decision and a lack of individualized care. A cultural difference between Canadian and European students' ethical thinking became apparent with such dilemmas as availability of abortion/ contraception services and women's right for abortion/ contraception. In particular, the Northern European students reflected uneasiness towards the cultural habit in Canada where special concern is given for the protection of patients' sexual integrity. The following quote from a Scandinavian student illustrates this cultural difference:

... annual appointment at a family doctor includes the PAP exam. If her family doctor was a male, it was astonishing that there had to be a female nurse present in the room during the gynaecological examination. It is a good thing to secure women's sexual integrity but this sounds as overprotection.... I cannot regard the doctor anything else but a professional person.

\section{Cultural and social differences}

In their reflections, the students documented an increased learning from these cultural and social differences that was either positive or negative in nature. Students' learning from the differences took place by witnessing practices on both ends of the continuum from effective/desirable to ineffective/undesirable. They reflected the unfamiliar cultural and social differences by comparing them with the familiar and thus began to understand the scope of primary health care in the host country. The reflected cultural and social differences were related to health 
programmes, services, techniques and methods, ranging from diverse ways of team work, uncommon aseptic and safety issues, to divergent or restricted nursing roles.

Aseptic and safety issues of the host health care were critiqued by both student groups. The European students were amazed how Canadian nurses threatened patient safety by wearing and cleaning their uniforms at home, by wearing the same shoes in and out of the hospital and by not maintaining good hand hygiene. The Canadian students witnessed in Europe an additional risk of injuries/ infection when handling contaminated needles and contaminated vesicles used for irrigation fluids. Home visits provided many opportunities to learn from cultural and social differences as documented by one Canadian student:

. . This particular event was based on a home visit to a rural family who has eight children in total, with their 2-year-old little boy who has a new diagnosis of asthma. . . . The main purpose of this visit was to do some asthma education with this family and when we were approaching the door we noticed that there was a baby carriage sitting on the front step. I thought it was just parked there, but then our preceptor peeked in and said that the baby is sleeping. I for sure was shocked, as we do not do this at home . . I am aware that I probably had a look of shock and awkwardness on my face when the father began setting out plates and cups for each of us, then making tea and coffee, bringing out cakes, cookies, and much more. . . . this is not normal in our culture, unless there are special circumstances; we are told not to accept anything from any of the clients that we visit.

\section{Health-care inequalities}

The students documented awareness and concern of health-care inequalities in their overseas reflections and examined host culture's health-care issues in the broader social, economic and political context. The reflections on health-care inequalities were related to accessibility and availability of services due to the political and cultural discrimination of minority groups, poor income, rural living and ageing. The students found that often the depriving determinants were bound together and resulted in poor health, poverty, social isolation and criminality.

Both student groups found health-care inequalities in the host country. In the rural communities, there were concerns about lack of available services and transporta-

10 tion to larger health-care facilities, increased demand on the district nurses' role and the impact of isolation and loneliness on the elderly. One European student reflected the inequality issue during the rural community placement in a school for aboriginal children in Canada as follows:

. . Many of the youngsters are seriously challenged in many segments of the determinant of health, low income, perhaps oppressive social environment and unfavourable genetics to biological factors. We of course cannot influence all of them but there are determinants that we can make a difference in a school setting. We can strengthen their individual coping skills. We can nurture their magnificently unique and beautiful culture and try to get them in touch with all of the values that have been the core stones of the way of life of their ancestors: wisdom, honesty, love, humanity, bravery, respect and truth.

\section{Population health concerns}

Students' reflections from the overseas experiences showed improved understanding of population health concerns in the host country and the cultural, social, economic and political relations. The students also realized the importance of community empowerment in health and well-being of people, as well as the need to change the focus of health services beyond curative care to health promotion. The students found that the major health concerns of western world include obesity, type 2 diabetes, cardiovascular diseases, mental disorders and substance abuse. Students noted these similar diseases on both sides of the Atlantic despite the long distance between the two continents.

Population health concerns were reflected by both the European and Canadian students but the European students visiting Canada witnessed even more complicated life situations presenting more diverse problematic health concerns than visa versa. The European students were faced with their own incapacity and emotional confusion in situations where unhealthy living habits, poverty, lack of social support and emotional problems were linked to health threats. A European student recounts the following poignant reflection:

. . The becoming mother did not have any supporting social network for obviously nobody knew about the father's existence and her parents had a severe alcohol problem. Both the mother and the baby also had so many health risks. . . . she used mild drugs, smoked a box of cigarettes in a day and had severe overweight. The girl also had mental problems and didn't rely

(C) 2009 Blackwell Publishing Asia Pty Ltd

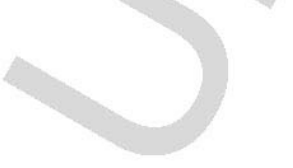


on the nurses and nutrition therapist and didn't emerge into the agreed appointments. The nurse said that the girl did not really understand that there was a human being growing inside of her body. . . . During the next meeting, the girl was more open and relaxed and spoke spontaneously. She told us that since the last visit she had not used drugs and smoked now only a small box of cigarettes. She had started to take the ordered vitamins and the agreed amount of vegetables and fruit beside all the garbage food. I had wanted to shake the girl and shout to her face whether she knew that she had the responsibility of somebody else's life.

\section{Personal and professional awareness}

Reflections from the trans-Atlantic experiences included documented evidence of an increase in students' personal and professional emotive self. This growth seemed to increase over time because of the adjustment of cultural differences and the additional communication with the host country. The students appreciated the unique experiences and recognized that the power of non-verbal communication in nursing care is transferable and portable for their future nursing experiences and careers. Achievement of these positive experiences was enabled when the students surpassed the hardships caused by the cultural differences and language barrier in daily life and nursing practice. Sometimes there were emotionally stressful situations where the students could not intervene verbally but it was noted that these experiences became pivotal both personally and professionally as 'the extreme, life-altering occurrences' (exchange student).

Both student groups encountered cultural and language barriers in their host country but the Canadian students were even more challenged by the use of non-verbal communication while leaning some basic words and phrases in the host language. Often such care situations were the most educational as documented by one Canadian student:

... a 7-year-old female who came into the clinic upon referral from a community health doctor to help diagnose Asperger's syndrome through observed characteristics in the home. Upon completion of each of these tasks, the child was diagnosed as not having Asperger's syndrome, but rather with having behavioural difficulties. We communicated very effectively, even with the language barrier. We were able to play

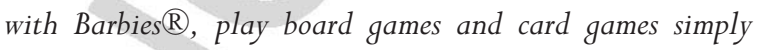
through acknowledging each other's tone of voice, hand gestures and simple Finnish words. I informed my nurse and other health-care staff, of what I noticed in her abilities and actions during playing and daily activities.

\section{DISCUSSION} Summary of the results

The enhancements of students' cultural competence were described through five main reflective learning categories: cross-cultural ethical issues, cultural and social differences, health-care inequalities, population health concerns and personal and professional awareness. The chosen critical incidents were contextually based experiences that the students identified as unfamiliar in comparing with the familiar; that either exemplified good healthcare practice or undesirable provision of care; or that profoundly affected them personally or professionally. The results of this research confirmed thee previous findings ${ }^{16,17}$ indicating a cross-cultural clinical and community experience as a process enhancing nursing students' personal and professional maturation, intellectual development and global perspective. Beneficial intercultural experiences include students' reflective process of uncomfortable, even shocking emotions arising from the cultural difference moving towards the development of a new perspective of understanding nursing care and the impact of diverse cultures in the surrounding world.

\section{Implications for nursing practice}

Collectively, student reflections suggest that the role of the health professional must extend into the health promotion realm, moving beyond its responsibility in only providing clinical and curative care. The experiences showed that the delivery of health services must embrace an expanded mandate that is sensitive and respects the diverse social and cultural aspects of people's lives. This mandate should support the needs of individuals and communities for a healthier lifestyle by creating channels between the health sector and broader social, political, economic and physical environmental components. A mandate that is consistent within a population health paradigm. Students noted that nurses need to learn to listen to the stories people tell them. They surmised if nurses listen to the cultural language of the local people and work collaboratively with them, they could develop long-term goals that were culturally congruent with the people they serve. Otherwise, it will become increasingly difficult to assist people to make a lifestyle change when the broader determinants of health are underdeveloped. 


\section{The CIT methodology}

The endeavour of this research project was to foster nursing students' cultural competence through a transformative learning process including critical reflection. CIT was used as a cultural learning method. Our results indicate that the CIT can promote students' cognitive and affective reflection skills during an international exchange and thus promote the development of a new cultural perspective for the health sector.

On the other hand there are personal and operational challenges ${ }^{18}$ that can inhibit cross-cultural reflection and development of cultural awareness during an international student exchange visit. The personal challenges emerge from students' immaturity, ethnocentrism and lack of self-regulation skills or inadequate language skills. The operational challenges include inadequate collaboration between the home and host institution, inadequate cultural orientation or debriefing, inadequate support in the host institution, lack of cultural immersion in the host culture or too short a time frame in the host country. This research project verified the importance of frequent and explicit dialogue between all partners during the course of the student exchanges. Despite common agreements and process guidelines, there should have been more reflections and discussions among the teaching staff about the academic supervision, tutorials and expected outcomes. The cultural orientation and preparation of the exchange students varied between the participating institutions and should have been more systematic and comprehensive. In particular, the Canadian students who had to bridge the language barrier during the stay in Estonia, Finland and Sweden should have been engaged in more profound cultural orientation. Finally, in a cross-cultural learning context, as described in this study, the students' academic reflective process should have been even more strongly supported by the local teaching staff. As Mezirow states in a dialogue with the educators, the learners justify their beliefs by giving and defending reasons and examining the evidence for and against the competing viewpoints. ${ }^{19}$

\section{Reliability concerns}

The assessment criteria of credibility, transferability, dependability and confirmability were used to confirm the trustworthiness of this research. ${ }^{20}$ The credibility of findings was established by: (i) prolonged engagement in the two-phased data collection; (ii) simultaneous and crisscross data analysis; and (iii) sharing the interpretations between the researchers. Transferability was enhanced by careful reporting of the research process, which allows the readers to judge the transferability and appropriateness of the findings in similar cross-cultural nursing education projects. Dependability was verified by constant comparison and contrasting of the data throughout the analysis process. Confirmability was verified by the maintenance of neutrality and prevention of personal bias in the research. These were controlled through a critical peer debriefing by the two principal researchers and the author peer review in the final phase of the writing process.

\section{CONCLUSION}

The CIT was successfully used to enhance nursing students' cultural reflection in this trans-Atlantic project. However, the following educational remarks should be noted to enhance the uptake of future CIT undertakings in the development of students' cultural competence as: (i) ensuring the learning objectives are shared between the project partners and students before they leave and throughout the exchange by explicitly articulating more discussion and support on 'what to expect'; and (ii) increasing dialogue either with the home or with the host instructor about each critical incident with specific learning and strategies for future experiences.

\section{ACKNOWLEDGEMENTS}

We would like to thank colleagues of the partner institutions in Finland, England, Estonia, Sweden, and Prince Edward Island, New Brunswick and Alberta, Canada for their contribution to this research project. Special thanks are due to the participating students who shared their cross-cultural critical reflections with us. We would like to express our gratitude to the European Union and the Government of Canada-Human Resources and Social Development Canada (HRSDC) for funding this transAtlantic student exchange programme between Europe and Canada.

\section{REFERENCES}

1 Koskinen L, Jokinen P. Multicultural health care. A transAtlantic project. Nurse Educator 2007; 32: 89-93.

2 O’Connor BB, Rockney R, Alario A. BaFá BaFáTM : A crosscultural simulation experience for medical educators and trainees. Medical Education 2002; 36: 1102.

3 Meleis AI. Culturally competent care. Journal of Transcultural Nursing 1999; 10: 12.

4 Koskinen L. [Gaining intercultural competence in a student exchange programme in nursing.] Journal of Nursing Science 2005; 17: 2-13 (in Finnish).

(C) 2009 Blackwell Publishing Asia Pty Ltd

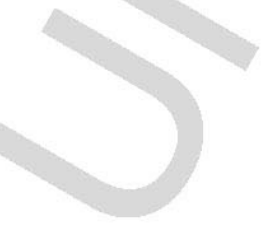

C2009 Blackwell Publishing Asia Pty Ltd 
5 Koskinen L, Abdelhamid P, Likitalo H. The simulation method for learning cultural awareness in nursing. Diversity in Health and Social Care 2008; 5: 55-63.

6 Bennett MJ. Towards ethnorelativism: A developmental model of intercultural sensitivity. In: Paige RM (ed.). Education for the Intercultural Experience. Yarmouth, ME, USA: Intercultural Press, 1993; 21-71.

7 Taylor EW. A learning model for becoming interculturally competent. International Journal of Intercultural Relations 1994; 18: 389-408.

8 The Ottawa Charter for Promotion. First International Conference on Health Promotion, Ottawa, 21 November 1986. Available from URL: http://wwwint/health promotion/conferences/previous/ottawa/en/index.html. Accessed $\bullet \cdot \cdots \cdot$

9 Flanagan J. The critical incident technique. Psychological Bulletin 1954; 51: 327-358.

10 Mikkonen I. Clinical learning as experienced by nursing students in their critical incidents (Academic dissertation). University of Joensuu. Publications in Education. No. 106, 2005.

11 Koskinen L, Jokinen P, Mikkonen I. [Critical incident technique as a method of learning reflection skills in nursing education.] Journal of Nursing Science 2007; 19: 90-101 (in Finnish).

12 Brookfield S. Using critical incidents to explore learners' assumptions. In: Mezirow J (ed.). Fostering Critical Reflection in Adulthood. A Guide to Transformative and Emancipatory Learning. San Francisco, CA, USA: Jossey-Bass, 1990; 177-193.

13 Luukka K. Meaningful learning experiences of the newly graduated practical nurses in the elderly care. Feeling mirror as a reflector of meaningful learning experiences (Academic dissertation). Kuopio University Publications E. Social Sciences 142, 2007 (in Finnish).

14 Redpath L, Stacey A, Pugh E, Holmes E. Use of the critical incident technique in primary care in the audit of deaths by suicide. Quality in Health Care 1997; 6: 25-28.

15 Miles MB, Huberman M. Qualitative Data Analysis. An Expanded Sourcebook. Newbury Park, CA, USA: Sage, 1994.

16 Thompson K, Boore J, Deeny P. A comparison of an international experience for nursing students in developed and developing countries. International Journal of Nursing Studies 2000; 37: 481-492.

17 Koskinen L, Tossavainen K. Study abroad as a process of learning intercultural competence in nursing. International Journal of Nursing Practice 2004; 10: 111-120.

18 Koskinen L. To survive you have to adjust. Study abroad as a process of learning intercultural competence in nursing (Academic dissertation). Kuopio University Publications E. Social Sciences 101, 2003

19 Mezirow J. Understanding transformation theory. Adult Education Quarterly 1994; 44: 222-232.

20 Lincoln YS, Guba EG. Naturalistic Inquiry. Beverly Hills, CA, USA: Sage, 1985. 


\begin{tabular}{|l|l|}
\hline \multicolumn{2}{|c|}{ SNP Best-set Typesetter Ltd. } \\
\hline Journal Code: IJN & Proofreader: Mony \\
\hline Article No: 1776 & Delivery date: 4 August 2009 \\
\hline Page Extent: 8 & Copyeditor: Harry \\
\hline
\end{tabular}

\section{AUTHOR QUERY FORM}

\section{Dear Author}

During the preparation of your manuscript, the questions listed below have arisen. Please answer all the queries (marking any other corrections on the proof enclosed) and return this form with your proofs.

\begin{tabular}{|c|c|c|}
\hline Query no. & Query & Reply \\
\hline q1 & AUTHOR: Please provide the position titles for all the authors. & \\
\hline$q^{2}$ & $\begin{array}{l}\text { AUTHOR: 'Université de Moncton' has been changed to 'University of Moncton'. Is this } \\
\text { OK? }\end{array}$ & \\
\hline $\mathrm{q}^{3}$ & AUTHOR: 'M.Sc.Inf.' has been changed to 'MScInf'. Is this OK? & \\
\hline q4 & $\begin{array}{l}\text { AUTHOR: 'Université de Moncton' has been changed to 'University of Moncton'. Is this } \\
\text { OK? }\end{array}$ & \\
\hline$q^{5}$ & $\begin{array}{l}\text { AUTHOR: As per journal style, the first-level headings in research papers include } \\
\text { INTRODUCTION, METHODS, RESULTS, DISCUSSION, ACKNOWLEDGEMENTS, } \\
\text { REFERENCES. Please change the headings of this paper as per journal style }\end{array}$ & \\
\hline q6 & $\begin{array}{l}\text { AUTHOR: '(CIT)' has been inserted here. In the following sentence, CIT's definition has } \\
\text { been deleted. Is this OK? }\end{array}$ & \\
\hline q7 & $\begin{array}{l}\text { AUTHOR: Please give manufacturer information for this product (Blackboard(C): company } \\
\text { name, town, state (if USA), and country }\end{array}$ & \\
\hline q8 & $\begin{array}{l}\text { AUTHOR: 'the students' documented' has been changed to 'the students documented'. Is } \\
\text { this OK? }\end{array}$ & \\
\hline q9 & AUTHOR: 'comparing them to' has been changed to 'comparing them with'. Is this OK? & \\
\hline q10 & AUTHOR: 'increase demand' has been changed to 'increased demand'. Is this OK? & \\
\hline q11 & $\begin{array}{l}\text { AUTHOR: 'comparing to the familiar' has been changed to 'comparing with the familiar'. } \\
\text { Is this OK? }\end{array}$ & \\
\hline q12 & $\begin{array}{l}\text { AUTHOR: A mandate that is consistent within a population health paradigm. The meaning } \\
\text { of this sentence is not clear; please rewrite or confirm that the sentence is correct }\end{array}$ & \\
\hline $\mathrm{m} 13$ & AUTHOR:: Please confirm Acknowledgements is correct. & \\
\hline $\mathrm{m} 14$ & $\begin{array}{l}\text { AUTHOR:: 'We would like to thank colleagues of the partner institutions in Finland, } \\
\text { England, Estonia, Sweden, Prince Edward Island, New Brunswick, and Alberta for } \\
\text { their..' has been changed to 'We would like to thank colleagues of the partner } \\
\text { institutions in Finland, England, Estonia, Sweden, and Prince Edward Island, New } \\
\text { Brunswick and Alberta, Canada for their. . .'. Is this OK? }\end{array}$ & \\
\hline q15 & $\begin{array}{l}\text { AUTHOR: Please provide the accessed date and please check this website address and } \\
\text { confirm that it is correct. (Please note that it is the responsibility of the author(s) to ensure } \\
\text { that all URLs given in this article are correct and useable.) }\end{array}$ & \\
\hline
\end{tabular}

